\title{
Hayabusa and its adventure around the tiny asteroid Itokawa
}

\author{
Makoto Yoshikawa, Akira Fujiwara, and Jun'ichiro Kawaguchi
}

ISAS/JAXA, 3-1-1 Yoshinodai, Sagamihara, Kanagawa 229-8510, Japan email: makoto@isas.jaxa.jp

\begin{abstract}
Results of the Hayabusa mission to image and sample the asteroid Itokawa are summarized.
\end{abstract}

Keywords. minor planets, asteroids, space vehicles

\section{Results}

The Hayabusa spacecraft, which was launched in May 2003, arrived at asteroid Itokawa in September, 2005. The size of Itokawa is about $540 \mathrm{~m}$ in length, so it is the smallest celestial object that manmade spacecraft has ever visited.

The view of Itokawa was totally unexpected. The surface of Itokawa is covered with numerous boulders and we could see only a few craters on it.

Hayabusa carried out scientific observations of Itokawa in detail for about two months by using four instruments; AMICA(Asteroid Multiband Imaging Camera), NIRS (NearInfrared spectrometer), XRS (X-ray fluorescence spectrometer), and LIDAR (Light Detection and Ranging instrument).

The surface of Itokawa is basically divided into two parts, smooth terrain and rough terrain, and we found many geographical and geological features form the images of AMICA with a resolution of less than $1 \mathrm{~cm}$ at the most. Also we found from the data of NIRS and XRS that the surface material of Itokawa is similar to LL-chondrite, ordinary chondrite meteorites.

The mass of Itokawa was estimated by the orbit analysis of Hayabusa using the optical navigation cameras, LIDAR, and radiometric data. Then the bulk density was calculated as $1.9 \mathrm{~g} / \mathrm{cm}^{3}$ using the obtained shape model of Itokawa. This low density indicates a macroporosity of about $40 \%$. From these results, we have concluded that Itokawa is a rubble pile asteroid.

After these scientific observations, Hayabusa tried to approach Itokawa closely several times in November 2005, and finally Hayabusa executed a touchdown on the surface of Itokawa twice. The second touchdown was executed as scheduled but after that we had troubles in the operation of Hayabusa. We are not sure if some surface materials were taken or not. However, expecting to have some samples, we are now attempting to return Hayabusa to the Earth in June 2010.

\section{Mission details}

Launch date: 9 May 2003

Arrival at Itokawa: 12 September 2005

Dry mass: $374 \mathrm{~kg}$, wet mass: $502 \mathrm{~kg}$ 
Primary science instruments:

Hayabusa-AMICA: collects scientific images with seven filters in the ECAS system bands: $u l, b, v, w, x, p$, and $z s$.

Hayabusa-LIDAR: uses time-of-flight measurement of a laser pulse reflected from the surface to determine the surface topography, with a footprint at $7 \mathrm{~km}$ altitude of about $5 \times 12 \mathrm{~m}$.

Hayabusa-NIRS: studies the mineralogy and physical properties of the surface with a 64 channel InGaAs photodiode array detector and a diffraction grating combined with a prism, covering wavelengths from 0.76 to $2.1 \mu \mathrm{m}$ with a dispersion per pixel of $23.6 \mathrm{~nm}$.

Hayabusa-XRs: studies the elemental composition of the surface using a CCD X-ray detector with an energy resolution of $160 \mathrm{eV}$ at $5.9 \mathrm{keV}$.

\section{In the literature}

A detailed description of the Hayabusa mission and the first results from the mission were published in the 2 June 2006 issue of Science. Fujiwara et al. (2006) give an overview of the rubble-pile asteroid Itokawa as observed by Hayabusa. M. Abe et al. (2006) discuss the near-infrared spectral results from the Hayabusa spacecraft. Okada et al. (2006) report on the X-ray fluorescence spectrometry by Hayabusa. Saito et al. (2006) present detailed images of asteroid 25143 Itokawa from Hayabusa. S. Abe et al. (2006) discuss mass and local topography measurements of Itokawa. Demura et al. (2006) report the pole and global shape of asteroide 25143 Itokawa. And Yano et al. (2006) describe the touchdown of the Hayabusa spacecraft at the Muses Sea on Itokawa.

\section{References}

Abe, M., Takagi, Y., Kitazato, K., Abe, S., Hiroi, T., Vilas, F., Clark, B. E., Abell, P. A., Lederer, S. M., Jarvis, K. S., Nimura, T., Ueda, Y., \& Fujiwara, A. 2006, Science, 312, 1334

Abe, S., Mukai, T., Hirata, N., Barnouin-Jha, O. S., Cheng, A. F., Demura, H., Gaskell, R. W., Hashimoto, T., Hiraoka, K., Honda, T., Kubota, T., Matsuoka, M., Mizuno, T., Nakamura, R., Scheeres, D. J., \& Yoshikawa, M. 2006, Science, 312, 1344

Demura, H., Kobayashi, S., Nemoto, E., Matsumoto, N., Furuya, M., Yukishita, A., Muranaka, N., Morita, H., Shirakawa, K., Maruya, M., Ohyama, H., Uo, M., Kubota, T., Hashimoto, T., Kawaguchi, J., Fujiwara, A., Saito, J., Sasaki, S., Miyamoto, H., \& Hirata, N. 2006, Science, 312, 1347

Fujiwara, A., Kawaguchi, J., Yeomans, D. K., Abe, M., Mukai, T., Okada, T., Saito, J., Yano, H., Yoshikawa, M., Scheeres, D. J., Barnouin-Jha, O. S., Cheng, A. F., Demura, H., Gaskell, R. W., Hirata, N., Ikeda, H., Kominato, T., Miyamoto, H., Nakamura, A. M., Nakamura, R., Sasaki, S., \& Uesugi, K. 2006, Science, 312, 1330

Okada, T., Shirai, K., Yamamoto, Y., Arai, T., Ogawa, K., Hosono, K., \& Kato, M. 2006, Science, 312, 1338

Saito, J., Miyamoto, H., Nakamura, R., Ishiguro, M., Michikami, T., Nakamura, A. M., Demura, H., Sasaki, S., Hirata, N., Honda, C.,. Yamamoto, A, Yokota, Y., Fuse, T., Yoshida, F., Tholen, D.J., Gaskell, R. W., Hashimoto, T., Kubota, T., Higuchi, Y., Nakamura, T., Smith, P., Hiraoka, K., Honda, T., Kobayashi, S., Furuya, M., Matsumoto, N., Nemoto, E., Yukishita, A., Kitazato, K., Dermawan, B., Sogame, A., Terazono, J., Shinohara, C., \& Akiyama H. 2006, Science, 312, 1341

Yano, H., Kubota, T., Miyamoto, H., Okada, T., Scheeres, D.J., Takagi, Y., Yoshida, K., Abe, M., Abe, S., Barnouin-Jha, O. S., Fujiwara, A., Hasegawa, S., Hashimoto, T., Ishiguro, M., Kato, M., Kawaguchi, J., Mukai, T., Saito, J., Sasaki, S., \& Yoshikawa, M. 2006, Science, 312, 1350 